\title{
Two-Photon Antenna Sensitization of Curium: Evidencing Metal-Driven Effects on Absorption Cross-Section in f-Element Complexes
}

Roger M. Pallares, ${ }^{1}$ Manuel Sturzbecher-Hoehne, ${ }^{1}$ Niranjan H. Shivaram, ${ }^{1,2}$ James P. Cryan, ${ }^{3,4}$ Anthony D’Aléo, ${ }^{5}$ and Rebecca J. Abergel ${ }^{1,6 *}$

${ }^{1}$ Chemical Sciences Division, Lawrence Berkeley National Laboratory, Berkeley, CA, 94720, USA

${ }^{2}$ Department of Physics and Astronomy, Purdue University, West Lafayette, IN 47907, USA

${ }^{3}$ Stanford PULSE Institute, SLAC National Accelerator Laboratory, 2575 Sand Hill Rd. Menlo Park, CA

${ }^{4}$ Linac Coherent Light Source, SLAC National Accelerator Laboratory, 2575 Sand Hill Rd. Menlo Park, CA

${ }^{5}$ Aix Marseille Université, CNRS, CINaM UMR 7325, Campus de Luminy, Case 913, 13288 Marseille, France

${ }^{6}$ Department of Nuclear Engineering, University of California, Berkeley, CA, 94720, USA *E-mail: abergel@,berkeley.edu

\section{TABLE OF CONTENTS}

Experimental Section. S2

Figure S1. Characterization of Tb(III)-3,4,3-LI(1,2-HOPO) for quantum yield determination. S4

Figure S2. Two-photon-excited fluorescence emission of fluorescein............................ S5

Figure S3. Emission spectrum of [Eu(III)-3,4,3-LI(1,2-HOPO)] .................................. S6

Figure S4. Emission spectrum of [Tb(III)-3,4,3-LI(1,2-HOPO)] ................................. S7

Figure S5. Emission spectrum of [Cm(III)-3,4,3-LI(1,2-HOPO)] ............................... S8

Figure S6. Emission spectrum of [Cm(III)-5-LIO(Me-3,2-HOPO)] ............................ S9

Figure S7. Emission spectrum of [Cm(III)-Enterobactin)] ...................................... S10

Table S1. Integrated ranges for two-photon absorption calculations.......................... S11

Supporting Information References........................................................................... S12 


\section{Experimental Section}

Caution: $\mathrm{Cm}^{3+}$ is radioactive and should be manipulated only in a specifically designated facility, in accordance with appropriate safety controls. All spectroscopic measurements were undertaken either in controlled facilities and/or using multiple containment procedures.

General. All chemicals were obtained from commercial suppliers and were used as received. The ligands 3,4,3-LI(1,2-HOPO) and 5-LIO(Me-3,2-HOPO) were synthesized by Ash Stevens, Inc. (Detroit, MI, USA) and AMRI, Inc. (Albany, NY, USA), respectively, following previously reported procedure, and used as received. ${ }^{1}$ Enterobactin was provided by Prof. K. N. Raymond (Department of Chemistry, University of California at Berkeley). The $\mathrm{LnCl}_{3} \cdot \mathrm{nH}_{2} \mathrm{O}$ lanthanide salts utilized were of the highest purity available $(>99.9 \%)$, and aliquots of acidified stocks of carrier-free ${ }^{248} \mathrm{Cm}\left(95.78 \%{ }^{248} \mathrm{Cm}, 4.12 \%{ }^{246} \mathrm{Cm}, 0.06 \%{ }^{245} \mathrm{Cm}\right.$, $0.02 \%{ }^{244} \mathrm{Cm} /{ }^{247} \mathrm{Cm}$ isotopic distribution by atom percentage) from the Lawrence Berkeley National Laboratory were used. All aqueous solutions were prepared using deionized water purified through a Millipore Milli-Q reverse osmosis cartridge system, and the $\mathrm{pH}$ was adjusted as needed with concentrated $\mathrm{HCl}$ or $\mathrm{KOH}$. Measurements were performed at room temperature

UV-Visible spectroscopy. UV-visible absorption spectra were recorded on an Ocean Optics USB 4000 spectrophotometer (slit $50 \mathrm{~mm}$, grating 600 grooves $/ \mathrm{mm}$, blaze $400 \mathrm{~nm}$ ) equipped with a DH2000-S light source, or on a NanoDrop 2000C, using quartz cells of $1.00 \mathrm{~cm}$ path length.

Fluorescence Spectroscopy. Emission spectra were recorded on a HORIBA Jobin Yvon IBH FluoroLog-3 spectrofluorimeter, used in steady state mode. A continuous xenon lamp (450 W) was used as the light source for single photon excitation. For two-photon excitation a tunable femtosecond Ti:Sapphire laser from Coherent (Chameleon Ultra) was used to excite over the $680-820 \mathrm{~nm}$ range with laser pulse duration of $140 \mathrm{fs}$, pulse repetition rate of 80 $\mathrm{MHz}$, focal length of lens of $10 \mathrm{~cm}$, and beam size of $1.2 \mathrm{~mm}$. A half-wave plate and polarized beam splitter acted as an attenuator and were placed in the beam path to control the laser beam power. A LabMax powermeter with PM10 sensor (Coherent) was used to determine the power before the beam entered the spectrofluorimeter. Emission was monitored perpendicular to the excitation pulse, with spectral selection achieved by passage through a double grating excitation monochromator $(2.1 \mathrm{~nm} / \mathrm{mm}$ dispersion, 1200 grooves $/ \mathrm{mm})$. A 
thermoelectrically cooled single photon detection module (HORIBA Jobin Yvon IBH, TBX04-D) was used to record spectra.

Quantum yield was determined with a FluoTime 300 (PicoQuant, Germany) and an integrating sphere accessory, following the fabricant directions. $100 \mu \mathrm{M} \mathrm{Tb}(\mathrm{III})-3,4,3-$ LI(1,2-HOPO) complex at pH $7.4(20 \mathrm{mM}$ HEPES and $20 \mathrm{mM} \mathrm{KCl})$ was used to quantify the quantum yield under $320 \mathrm{~nm}$ excitation in a quartz cuvette (5mm path length) (Figure S1).

Ligand and Metal working Solutions. The $\mathrm{Eu}(\mathrm{III}), \mathrm{Tb}(\mathrm{III})$, and $\mathrm{Cm}(\mathrm{III})$ working stock solutions were prepared in standardized 0.1 M HCl. Aqueous stock solutions of 3,4,3-LI(1,2HOPO) and 5-LIO(Me-3,2-HOPO) were freshly prepared by direct dissolution of a weighed portion of ligand in purified Milli-Q water prior to each set of experiments. An Ent stock solution was prepared in DMSO and diluted prior to the experiment in deionized water. The $\mathrm{pH}$ of the $100 \mathrm{mM}$ HEPES buffer was adjusted at 7.4 and the buffer solution diluted 2-fold in final sample solutions. All metal-complex solutions had a stoichiometry of 1 : 1 metal : ligand.

Data Processing and Calculations. The formula used to calculate the two-photon absorption cross-sections was adapted from Albota et al. ${ }^{2}$

$$
\sigma_{2, \text { new }}=\frac{\sigma_{2, \text { ref }} \cdot \Phi_{\text {ref }} \cdot I_{\text {new }} \cdot P^{2}{ }_{\text {ref }} \cdot c_{\text {ref }}}{\Phi_{\text {new }} \cdot I_{\text {ref }} \cdot P^{2}{ }_{\text {new }} \cdot c_{\text {new }} \cdot A_{\text {int new }}}
$$

Where $\sigma_{2}$ is the cross-section in Goeppert-Mayer units (GM) of the new analyte (new) or the reference (ref), $\Phi$ is the quantum yield, $P$ is the laser power at the specific $\lambda, I$ is the integrated emission intensity at the specific $P, c$ is the concentration of the new analyte or reference, and $A_{\text {int }}$ is the fraction of integrated emission area. $10 \mu \mathrm{M}$ fluorescein in $\mathrm{H}_{2} \mathrm{O}(\mathrm{pH}$ 11) with 17 GM cross-section ${ }^{3}$ was used as reference (Figure S2).

Only a fraction of the emission spectra of the complexes could be recorded under two-photon excitation to calculate the area. Hence, the area values were corrected (as described in the previous paragraph) to account for the total emission area sensitized by the ligands. Figures $\mathrm{S} 3$ to $\mathrm{S} 7$ plot the whole emission spectra of the complexes under single-photon excitation, highlighting the range recorded in the two-photon experiments and reporting the percentage that the range represents from the total emission area. These values are summarized in Table S1. 

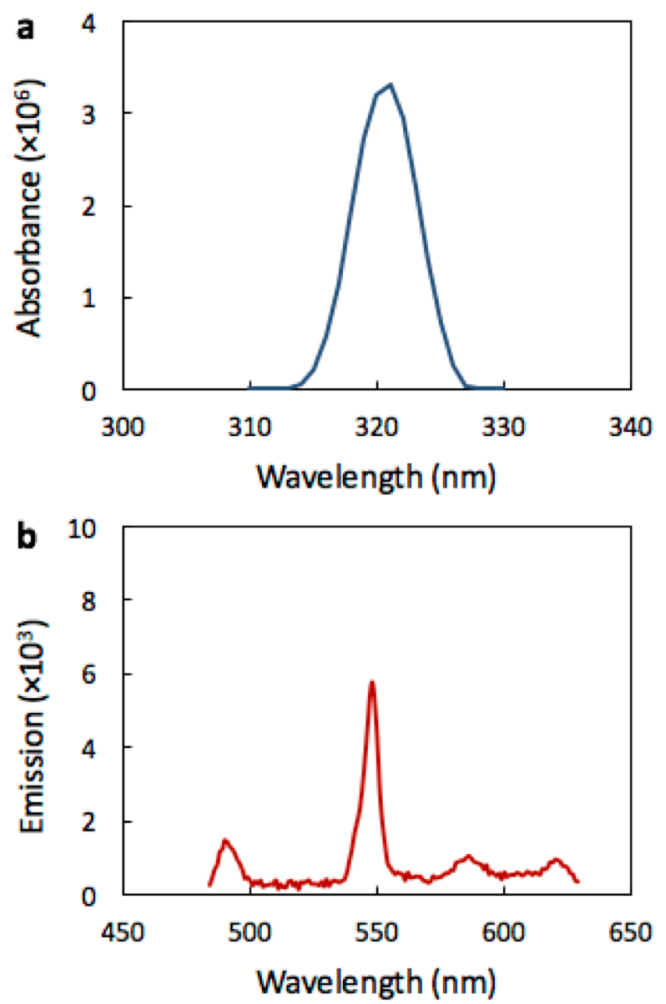

Figure S1. Characterization of Tb(III)-3,4,3-LI(1,2-HOPO) for quantum yield determination. (a) Absorbance and (b) emission of $100 \mu \mathrm{M}$ Tb(III)-3,4,3-LI(1,2-HOPO) at $\mathrm{pH} 7.4(20 \mathrm{mM}$ HEPES and $20 \mathrm{mM} \mathrm{KCl})$ under excitation at $320 \mathrm{~nm}$. 



Figure S2. Two-photon-excited fluorescence emission of fluorescein. (a) Emission spectra of $10 \mu \mathrm{M}$ fluorescein in $\mathrm{H}_{2} \mathrm{O}(\mathrm{pH} \mathrm{11})$ under excitation at $684 \mathrm{~nm}$. The laser power varies between $75-1220 \mathrm{~mW}$. The integrated area was $470-620 \mathrm{~nm}$ corresponding to $100 \%$ of the total area sensitized. (b) Spectral change of fluorescein with increased laser power. 


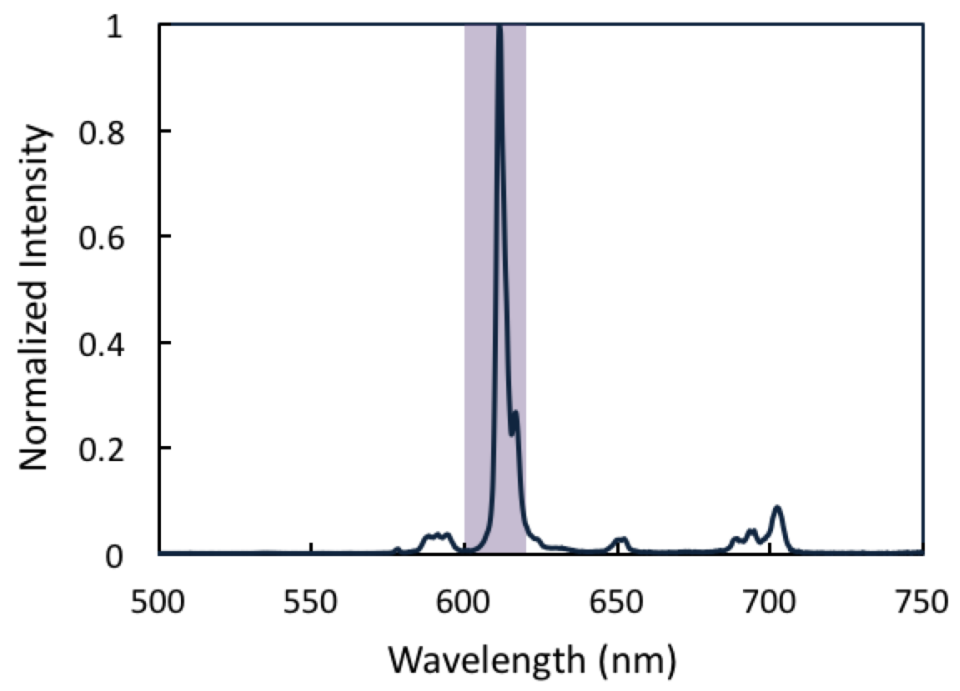

Figure S3. Emission spectrum of [Eu(III)-3,4,3-LI(1,2-HOPO)]. The integrated area is highlighted in purple color, which represents $79 \%$ of the spectrum. The emission was recorded under $320 \mathrm{~nm}$ excitation. 


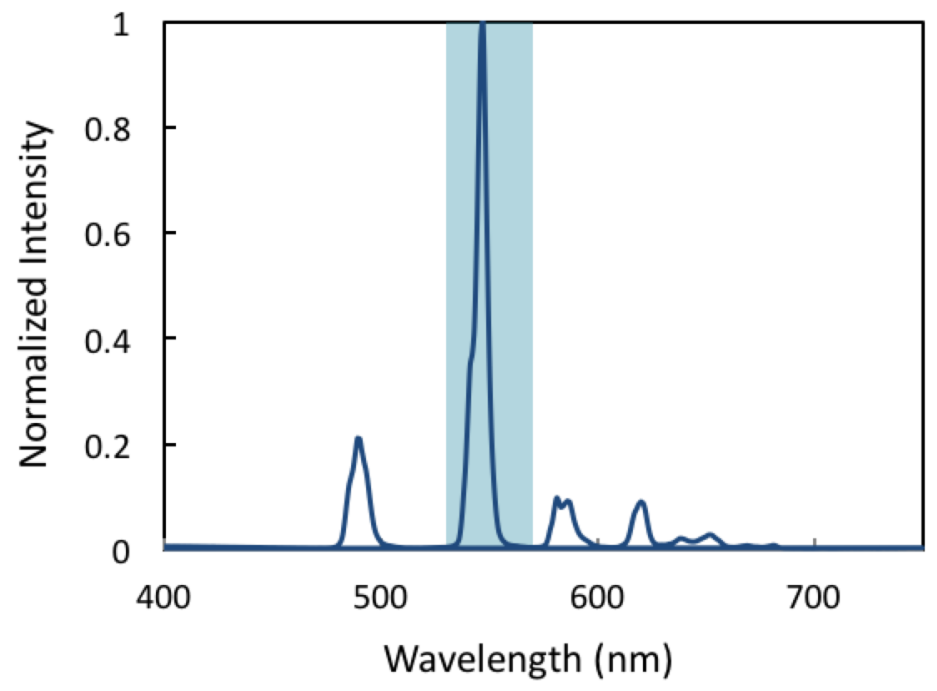

Figure S4. Emission spectrum of [Tb(III)-3,4,3-LI(1,2-HOPO)]. The integrated area is highlighted in blue color, which represents $59 \%$ of the spectrum. The emission was recorded under $320 \mathrm{~nm}$ excitation. 


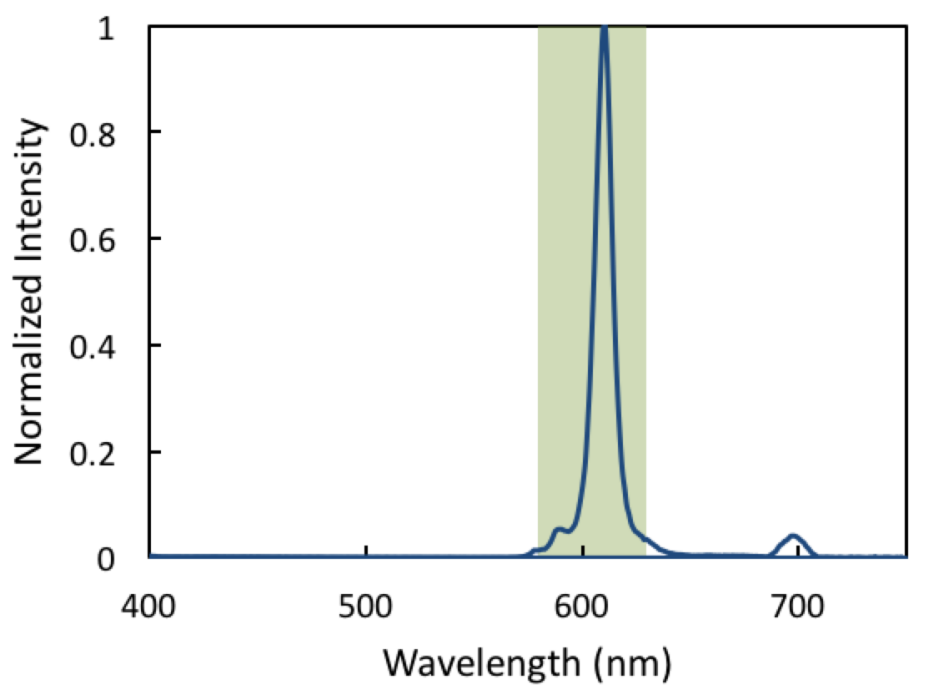

Figure S5. Emission spectrum of [Cm(III)-3,4,3-LI (1,2-HOPO)]. The integrated area is highlighted in green color, which represents $92 \%$ of the spectrum. The emission was recorded under $320 \mathrm{~nm}$ excitation. 


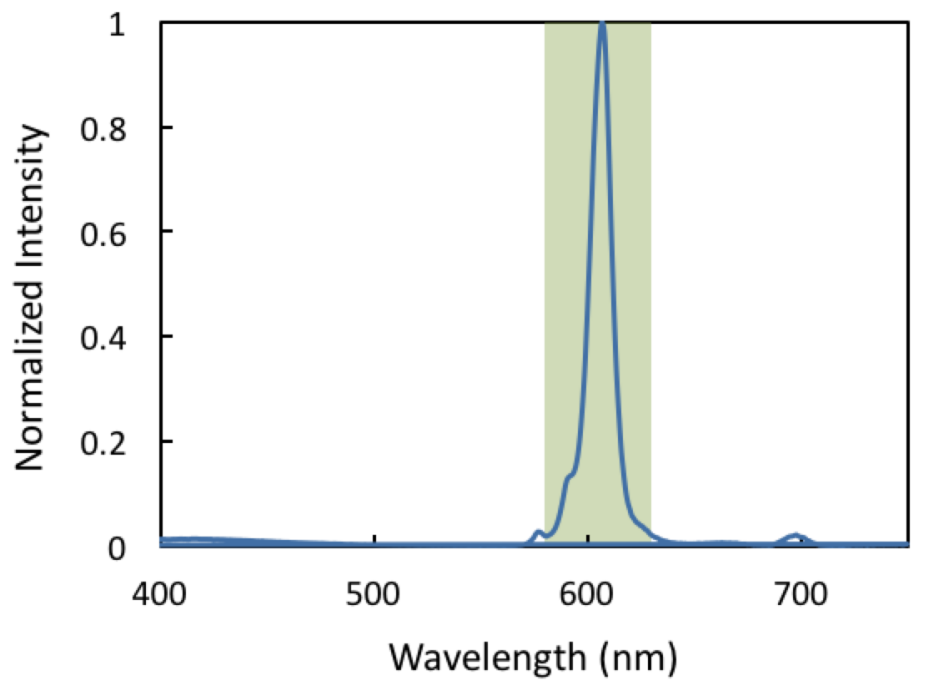

Figure S6. Emission spectrum of [Cm(III)-5-LIO(Me-3,2-HOPO)]. The integrated area is highlighted in green color, which represents $90 \%$ of the spectrum. The emission was recorded under $345 \mathrm{~nm}$ excitation. 


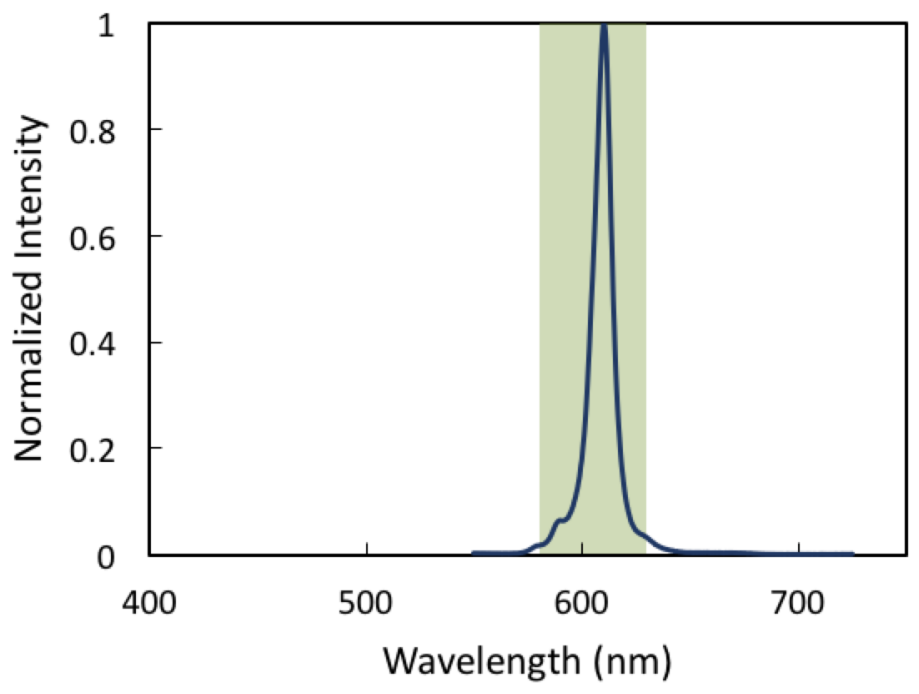

Figure S7. Emission spectrum of [Cm(III)-Enterobactin)]. The integrated area is highlighted in green color, which represents $96 \%$ of the spectrum. The emission was recorded under $345 \mathrm{~nm}$ excitation. 
Table S1. Integrated ranges for two-photon absorption calculations

\begin{tabular}{cccc}
\hline Complexes & Integrated range & \% of the total area & Figure \\
\hline $\mathrm{Eu}(\mathrm{III})-3,4,3-\mathrm{LI}(1,2-\mathrm{HOPO})$ & $600-620 \mathrm{~nm}$ & 72 & $\mathrm{~S} 3$ \\
$\mathrm{~Tb}(\mathrm{III})-3,4,3-\mathrm{LI}(1,2-\mathrm{HOPO})$ & $530-570 \mathrm{~nm}$ & 59 & $\mathrm{~S} 4$ \\
$\mathrm{Cm}(\mathrm{III})-3,4,3-\mathrm{LI}(1,2-\mathrm{HOPO})$ & $580-630 \mathrm{~nm}$ & 92 & $\mathrm{~S} 5$ \\
$\mathrm{Cm}(\mathrm{III})-5-\mathrm{LIO}(\mathrm{Me}-3,2-\mathrm{HOPO})$ & $580-630 \mathrm{~nm}$ & 90 & $\mathrm{~S} 6$ \\
$\mathrm{Cm}(\mathrm{III})-$ enterobactin & $580-630 \mathrm{~nm}$ & 96 & $\mathrm{~S} 7$ \\
\hline
\end{tabular}




\section{Supporting Information References}

(1) Abergel, R. J.; Durbin, P. W.; Kullgren, B.; Ebbe, S. N.; Xu, J.; Chang, P. Y.; Bunin, D. I.; Blakely, E. A.; Bjornstad, K. A.; Rosen, C. J.; Shuh, D. K.; Raymond, K. N. Biomimetic actinide chelators: an update on the preclinical development of the orally active hydroxypyridonate decorporation agents 3,4,3-LI(1,2-HOPO) and 5-LIO(Me-3,2-HOPO). Health Phys. 2010, 99 (3), 401-407.

(2) Albota, M. A.; Xu, C.; Webb, W. W. Two-photon fluorescence excitation cross sections of biomolecular probes from 690 to $960 \mathrm{~nm}$. Appl. Opt. 1998, 37 (31), 7352-7356.

(3) Makarov, N. S.; Drobizhev, M.; Rebane, A. Two-photon absorption standards in the 550-1600 nm excitation wavelength range. Opt. Express 2008, 16 (6), 4029-4047. 